\title{
Anti-CD73 Monoclonal Antibody CPI-006
}

National Cancer Institute

\section{Source}

National Cancer Institute. Anti-CD73 Monoclonal Antibody CPI-006. NCI Thesaurus. Code C156061.

A type II humanized immunoglobulin G1 (IgG1) monoclonal antibody targ eting the ectoenzyme 5'-ecto-nucleotidase (cluster of differentiation 73; CD73; 5'-NT; ecto-5'nucleotidase; NT5E), with potential immunomodulating and antineoplastic activities. Upon intravenous administration, anti-CD73 monoclonal antibody CPI-006 targets and binds to CD73 on tumor cells, leading to internalization of CD73. This prevents CD73mediated conversion of extracellular adenosine monophosphate (AMP) to adenosine, thereby preventing adenosine-mediated suppression of lymphocyte activity and increasing the activity of cytotoxic T-lymphocytes (CTLs). This also activates macrophages, and reduces the activity of both myeloid-derived suppressor cells (MDSCs) and regulatory $\mathrm{T}$-lymphocytes. By abrogating the inhibitory effect on the immune system and enhancing the CTL-mediated immune response against cancer cells, tumor cell growth is decreased. CD73, a plasma membrane protein belonging to the 5'nucleotidase (NT ase) family, is upregulated on a number of cancer cell types and catalyzes the conversion of extracellular nucleotides, such as AMP, to membranepermeable nucleosides, such as adenosine; it plays a key role in adenosine-mediated immunosuppression within the tumor microenvironment. 